\title{
TRANSTRAÇÃO E TRANSPRESSÃO AO LONGO DO LINEAMENTO CINZENTO (REGIÃO DA SERRA DOS CARAJÁS)
}

\author{
JOÃO BATISTA SENA COSTA* e JOSÉ BATISTA SIQUEIRA**
}

\begin{abstract}
TRANSTENSION AND TRANSPRESSION ALONG THE CINZENTO LINEAMENT. The sinistrai strike-slip Cinzento lineament is formed by compressional and extensional segments and represents a small fraction of the Itacaiunas shear belt. From west to east these structures are identified as the Igarapé Salobo extensional duplex, the Curuni compressional duplex and the Serra Pelada compressional horsetail. The eliptical geometry of the Igarapé Salobo structure is defined by a major normal shear zone mat converge to the E-W strike-slip detachment zone; the internal geometry consists of a set of NW-SE oblique normal Tones. Its kinematic evolution includes transtension followed by transpression and then transtension. The Cururu duplex is a rhomboedric structure related to E-W Y or D zones and NW-SE P zones interaction. Its internal geometry comprises oblique thrust zones linked to the major strike-slip zones, and its kinematic evolution involves transtension followed by transpression and strike-slip displacements. The Serra Pelada horse-tail includes NE-SW oblique thrust zone wich diverge from the master E-W strike-slip zone. Its kinematic history includes transtension, transpression and displacements in $\mathrm{R}^{*}$ zones. Important deposits of gold, sulphides, rock crystal and amethyst are associated to the Cinzento lineament evolution.
\end{abstract}

Keywords: Compressive strik-slip duplex, horse-tail structure, extensional strike-slip duplex.

\begin{abstract}
RESUMO O Lineamento Cinzento é formado por vários feixes de zonas de cisalhamento sinistrais e constitui uma pequena fração da evolução do Cinturão Itacaiunas no final do Arqueano. Ao longo desse lineamento reconhecem-se duplexes compressivos e distensivos e rabo de cavalo, que experimentaram transtracão, transpressão e deslocamentos transcorrentes na progressão da deformação. Tais estruturas são identificadas por meio da seguinte nomenclatura: duplex distensivo Igarapé Salobo, duplex compressivo Cururu e rabo de cavalo compressivo Serra Pelada. O duplex distensivo Igarapé Salobo tem forma elfptica e sua geometria é definida por uma zona de deslocamento normal que converge para a zona transcorrente E-W do sistema principal. Internamente, as zonas normais secundárias têm orientação NW-SE; sua história cinemática inclui, transtraçab inicial, seguida por transpressão e transtração final. $\mathrm{O}$ duplex compressivo Cururu é uma estrutura romboédrica formada pela articulação de zonas transcorrentes Y ou D (E-W) e P (NW-SE); a geometria interna compreende zonas de cavalgamento que se ajustam ás zonas transcorrentes principais e a evolução é entendida a partir de transtração seguida por transpressão e por movimentação essencialmente direciona!. O rabo de cavalo compressivo Serra Pelada é alongado na direção NE-SW, seguindo a orientação das zonas de cavalgamento secundárias que divergem a partir da zona mestra transcorrente (E-W); sua evolução cinemática envolve transtração, transpressão e deslocamento ao longo de zonas R'. Importantes depósitos de ouro, sulfetos, cristal de rocha e ametista estão associados à evolução do Lineamento Cinzento.
\end{abstract}

Palavras-chaves: Duplex direciona! compressivo, estrutura em rabo-de-cavalo, duplex direciona! distensivo.

INTRODUCÃ̃O Os ingredientes básicos da tectônica transcorrente foram visualizados há algum tempo (Wilcox et al. 1973, CroweH 1974, Oxburgh 1974, Balance \& Reading 1980, Badham 1982, Dewey 1982), mas só começaram a ser amplamente discutidos recentemente (Sanderson \& Marchini 1984, Buck \& Biddle 1985, Woodcock \& Fischer 1986, Woodcock 1986 etc).

A aplicação desse conjunto de conhecimentos ainda é precária no contexto dos terrenos pré-cambrianos, em particular no Arqueano. Na Amazônia Oriental, Araújo et al. (1988) e Souza et al. (1988) demonstraram a importância do sistema transcorrente na evolução de cinturões de cisalhamento oblíquo e de terrenos granito-greenstone respectivamente, a partir da caracterização de estruturas-em-flor que experimentaram transtração e transpressão.

Este artigo discute a geometria e a cinemática do Lineamento Cinzento localizado na parte norte da Serra dos Carajás, no sudeste do Estado do Pará (Figs, 1 e 2). Como se trata de um exemplo antigo (Arqueano) e didático, julgou-se oportuno divulgar os resultados preliminares das investigações que os autores estão deșenvolvendo em diferentes regiões ao longo do lineamento. É importante enfatizar que a caracterização das diferentes estruturas que compõem $\mathrm{p}$ lineamento proporcionou o entendimento dos depósitos minerais conhecidos na região. Tais estruturas são consideradas hoje como armadilhas estruturais que serviram de condutos para percolação de fluidos.

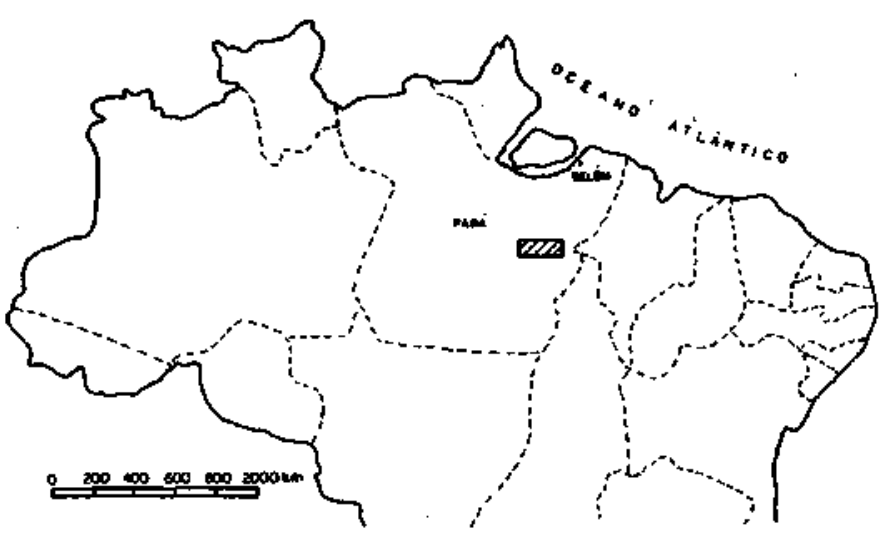

$\mathbb{Z Z}$

Figura I - Mapa de localizaçoo da drea Fitgure L - Location map of study aros

\footnotetext{
* Departamento de Geologia, Centro de Geociências, Universidade Federal do Pará, Caixa Postal 1611, CEP 66050, Belém, Pará, Brasil

** Engenharia e Consultoria S/A, ENGE-RIO, Travessa Lomas Valentines, 2717 - Marco, CEP 66240, Belém, Pará, Brasil
} 


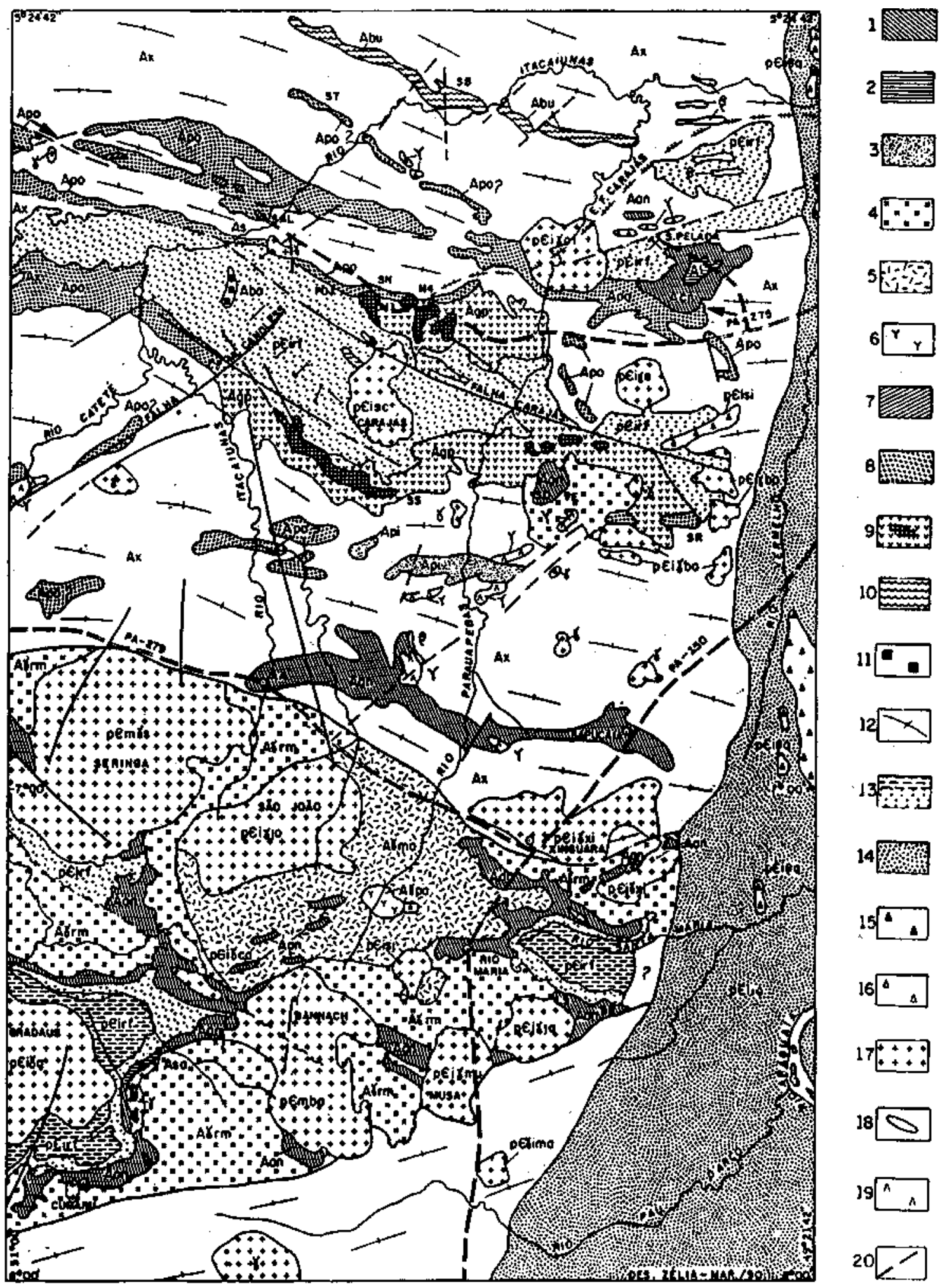

Figura 2 - Mapa Geológico Regional - Província Mineral de Carajás. 1. Supergrupo Andorinhas (Aon); 2. Complexos diferenciados Luanga (AI) e Serra Azul (Asa); 3. Complexo Pium (Api); 4. Granodiorito Rio Maria (Ayrm); 5. Trondhjemito Mogno (Aymo); 6. Tonalito Parazônia (Aypa); 7. Grupo Igarapé Salobo (As); 8. Grupo Igarapé Pojuca (Apo); 9. Grupo Grão Pará (Agp); 10. Grupo Buritirama (Abu); 11. Grupo Igarapé Bahia (Aba); 12. Complexo Xingu (Ax); 13. Grupo Rio Fresco (peirf); 14. Grupo Tocantins (peito); 15. Suite ultramáfica Quatipuru (peifip); 16. Gabro Santa Inês (peisi); 17. Granitos anorogênicos (peiy: ei - cigano, se - Carajás, mu - Musa, g-Gradaus, bo - borrachudo, xi - xinguara, sj - São José, ma - Marajoara, e - Estrela, ba - Bannach pemy, se-Seringa, já - Jamon); 18. Diques de diabásio; 19. Rochas ultramáficas; 20. Falha. Localidades: SAL. Salobo; NI. Acampamento NI; POJ. Acampamento Pojuca; AA. Água Azul; N4 - Jazida de ferro; BB. Babaçu; LS. Lagoa Seca; SD. Serra Dourada; SN. Serra Norte; SS. Serra Sul; ST. Serra Tapirapé; SR. Serra do Rabo; VE. Rocha Ultrabásica do Vermelho; SB. Serra do Buritirama; Região compreendida pelo Lineamento Cinzento

Figure 2 - Geological map of the Carajás Mineral Province 
GEOMETRIA O Lineamento Cinzento é formado principalmente por dois duplexes (duplex distensivo Igarapé Salobo e duplex compressivo Cururu) e por um rabo de cavalo (rabo de cavalo compressivo Serra Pelada), separados por segmentos retos (Fig. 3). Neste item, são mostrados os principais aspectos geométricos dessas estruturas.

Duplex distensivo Igarapé Salobo O duplex distensivo Igarapé Salobo encontra-se na extremidade oeste do Lineamento Cinzento e é composto principalmente por quartzitos, formações ferríferas, xistos aluminosos, xistos com magnetita, xistos quartzo- feldspáticos e frações de gnaisses tonalíticos e graníticos. Tais conjuntos rochosos ocorrem sob a forma de faixas alongadas na direção NW-SE e seus contatos são, em geral, definidos por meio de zonas de concentração de movimentação ou zonas de cisalhamento. Ao longo das zonas de cisalhamento há registros de transformações mineralógicas em fácies anfibolito, superpostas por assembléias mineralógicas da fácies xisto verde. Os diferentes termos petrográficos acham-se desfeitos em lentes, compondo um padrão geométrico fortemente anastomosado.

A forma geral do duplex assemelha-se a uma estrutura elíptica assimétrica, orientada na direção $\mathrm{E}-\mathrm{W}$ e definida por uma zona de cisalhamento transcorrente sinistrai e por um zona de cisalhamento normal oblíqua curvilínea, que converge para a zona transcorrente. Internamente, a estrutura é composta por zonas de cisalhamento normais secundárias, orientadas preferencialmente na direção NW-SE, que se ligam às zonas principais normal e transcorrente em direção a NW e SE, respectivamente. $\mathrm{O}$ mapa da figura 3 mostra a forma geral do duplex e as figuras $4 \mathrm{a}$ e $4 \mathrm{~b}$ representam as prováveis interpretações tridimensionais. Vê-se, portanto, que se trata de uma estrutura assimétrica, com zonas secundárias convergindo para o deslocamento transcorrente (Fig. 4a), ou para o deslocamento normal oblíquo (Fig. 4b). Na ausência de informações de subsuperfície, as duas interpretações geométricas são consideradas neste trabalho.

As zonas de cisalhamento são caracterizadas internamente por uma foliação milonítica penetrativa que se assemelha a uma foliação de transposição e, localmente, dá lugar a um bandamento composicional. Essa foliação, classificada como $S$, é seccionada, deslocada e encurvada pela foliação $C$, proporcionando a definição do sentido de movimentação das zonas de cisalhamento. Nos locais onde a deformação foi mais intensa, as foliações $\mathrm{S}$ e $\mathrm{C}$ encontram-se paralelizadas, contribuindo para o desenvolvimento do padrão lenticularizado. Bandas de cisalhamento centimétricas a decimétricas também são vistas localmente e ajudaram na caracterização do sentido de transporte das massas rochosas. A lineação de estiramento, presente no plano de foliação milonítica que define as zonas secundárias, tem orientação NW-SE, mergulha fortemente para SE e é formada principalmente por barras de quartzo, bem como por frações lenticulares dos diferentes conjuntos litológicos. Ao longo da zona transcorrente principal a lineação de estiramento orienta-se na direção WNW-ESE e mergulha suavemente para ESE, sugerindo que essa zona também incorpora uma componente de mergulho normal.

Essa geometria é localmente modificada por zonas de cisalhamento transcorrentes secundárias dextrais e sinistrais com direções NNE-SSW e NW-SE, respectivamente.

Além das estruturas mencionadas, há, localmente, registros de foliações e lineações minerais e de estiramento formada sob condições de fácies anfibolito. Tais estruturas devem estar ligadas a um movimento cinemático anterior ao desenvolvimento do duplex distensivo.

Duplex compressivo Cururu $\mathrm{O}$ duplex compressivo $\mathrm{Cu}-$ ruru é composto essencialmente por metarenitos, quartzitos, formações ferríferas, rochas metabásicas, xistos e gnaisses subordinados, compondo faixas orientadas na direção NW-SE, com variações para E-W, moldando-se à geometria geral das zonas de cisalhamento que formam a estrutura maior. Ao longo das zonas de cisalhamento, os diversos termos petrográficos são transformados em brechas com fragmentos que podem alcançar dimensões deciméíricas, cataclasitos, protomilonitos e milonitos. Em geral, as assembléias mineralógicas iden-

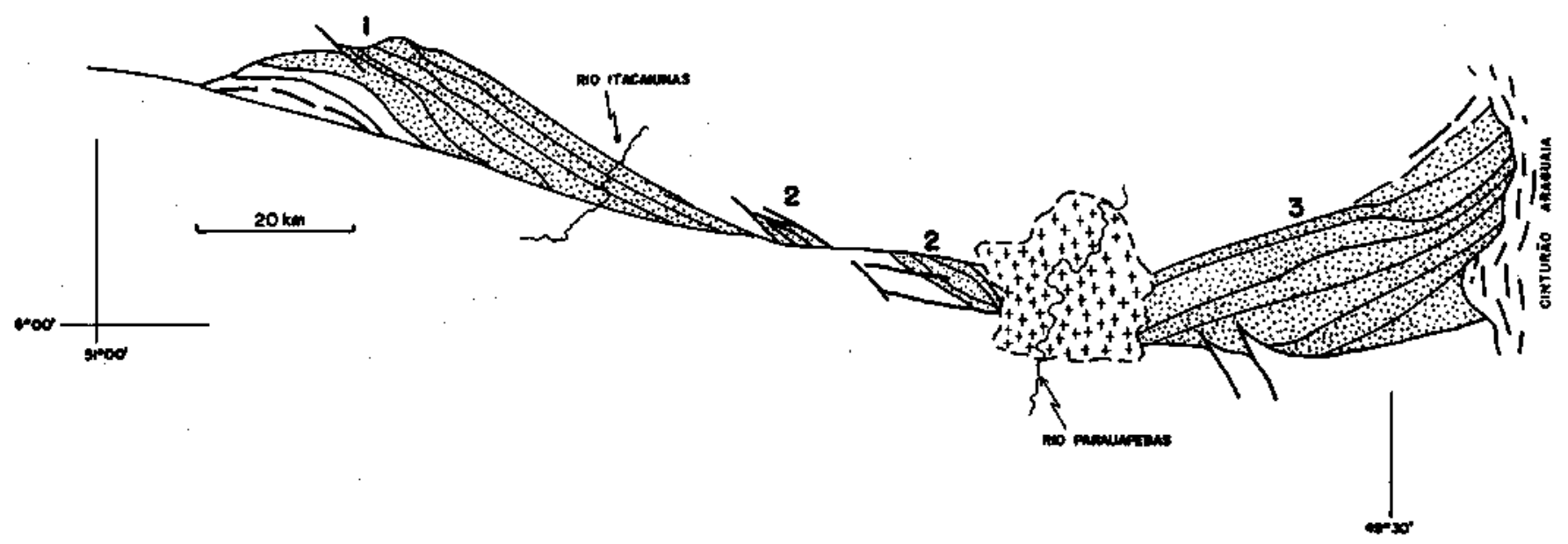

Figura 3 - Esboço litoestrutural simplificado do Lineamento Cinzento: 1. duplex distensivo Salobo; 2. duplex compressivo Cururu; 3. rabo de cavalo Serra Pelada. Em branco: complexo gnáissico-granitóide. Pontilhado: unidades de rochas supracrustais ligadas a evolução do Lineamento Cinzento. Tracejado: unidades de rochas supracrustais do Cinturão Araguaia. Cruzes: Granito Cigano do Proterozóico Médio

Figure 3 - Sketch map pf the Cinzento Lineament. 1. Salobo extensional duplex; 2. Cururn compressive duplex; 3. Serra Pelada horsetail. Blankgneissic complex; dotted-supracrustal rocks related to the Cinzento Lineament evolution; dashed-supracrustal rocks of the Araguaia belt; crosses-Cigano granite of Middle Proterozoic 
tificadas são compatíveis com as condições térmicas da fácies xisto verde baixa a média. Trata-se de uma estrutura romboédríca alongada na direção NW-SE, formada pela interação entre zonas de cisalhamento $P$ e $Y / D$ e seccionada em dois segmentos por uma zona de cisalhamento $Y / D$ na progressão da deformação (Fig. 3 e 5). Sua geometria interna é formada por zonas de cisalhamento curvilíneas secundárias, com caráter de cavalgamento e arranjadas de modo a definir uma estrutura divergente positiva e simétrica. A figura 6 mostra a geometria das zonas de cisalhamento secundárias em profundidade e suas relações espaciais com as zonas transcorrentes principais.
A

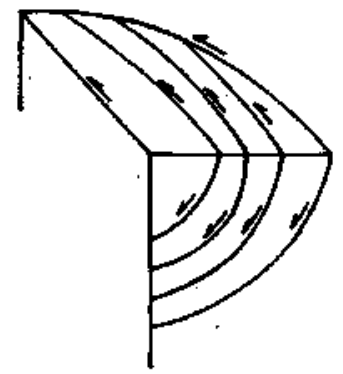

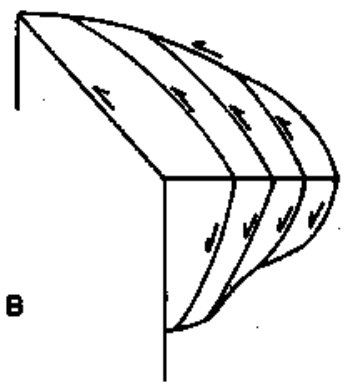

Figura 4-Blocos diagramas mostrando os arranjos geométricos visualizados para o duplex distensivo Salobo: A. as zonas de cisalhamento secundárias se ajustam ao plano de deslocamento transcorrente subvertical; B. as zonas de cisalhamento secundárias convergem para um plano de deslocamento basal sub-horizontal. Os planos frontais dos blocos diagramas representam seções $\mathrm{N}-\mathrm{S}$ na parte central do duplex (Fig. 3), com $o$ observador olhando para oeste. Sem escala Figure 4 - Block diagrams showing the idealized geometric pattern of the Salobo extensional duplex: A. minor shear zones linked to the steep strike-slip detachment zone; B. minor shear zones converge to low angle detachment zone. The frontal planes of the block diagrams represent N-S sections in the central part of the duplex (Fig. 3), with the observer feeing West. Without scale

As zonas de cisalhamento são caracterizadas por urna foliação milonítica paralela ao bandamento composicional definido por frações, regulares ou não, de rochas corríposicionalmente distintas. Localmente, vê-se que essa foliação encontra-se superposta pela foliação $C$, e por bandas de cisalhamento, bem como por zonas de cisalhamento decimétricas $Y / D$, contribuindo pára a definição do arranjo anastomosado ou lenticularizado. A lineação de estiramento orienta-se nas direções E-W e NE-SW, ao longo das zonas transcorrentes e secundárias respectivamente, e, em geral, é definida por bastões de quartzo.

Zonas de cisalhamento na direção NE-SW, em geral, são preenchidas por material silicoso e interpretadas como zonas $T$ e $R^{\prime}$, também superpõem-se à arquitetura principal do duplex.

Rabo de Cavalo Compressivo Serra Pelada O rabo de cavalo compressivo Serra Pelada corresponde à extremidade leste do Lineamento Cinzento e é composto por zonas de cavalgamento NE-SW que divergem da zona transcorrente principal, orientada na direção E-W; sua constituição litológica inclui arenitos, siltitos e formações ferríferas, além de rochas vulcânicas e plutônicas máficas e ultramáficas, que se encontram transformadas em metamorfitos de fácies xisto verde ao longo das zonas de cisalhamento.

As zonas de cisalhamento são definidas pela presença de uma foliação milonítica fortemente inclinada para sudeste que, dependendo da natureza da rocha envolvida, pode ser classificada como xistosidade ou clivagem de fratura. $\mathrm{O}$ bandamento

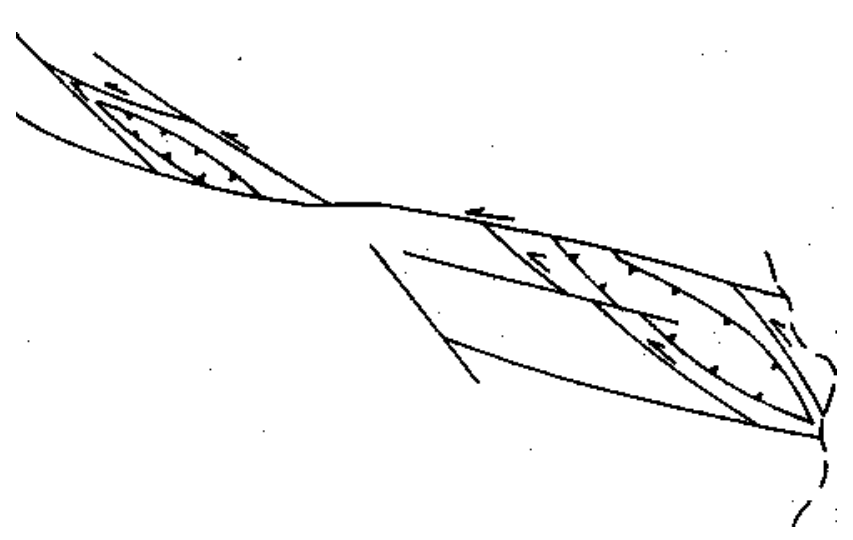

Figura 5 - Esboço litoestrutural do duplex Cururu. Em branco: complexo gnáissico-granitóide. Pontilhado: rochas supracrustais. Para localização, ver figura 3. Sem escala Figure 5- Litho-structural sketch of the Cururu duplex. Blank-gneissic complex; dotted-supracrustal rocks. Without scale

composicional é paralelo à foliação milonítica. A lineação de estiramento nas zonas de cavalgamento tem direção NE-SW e mergulha em média $20^{\circ}$ para SW, mas, nas zonas transcorrentes, sua orientação é E-W. Os critérios de rotação analisados indicam movimentação essencialmente sinistrai na evolução desse sistema. Vê-se na figura 7 que se trata de uma estrutura assimétrica positiva e que as zonas secundárias podem ter evoluído a partir de um cavalgamento basal (Fig. Tb) ou do deslocamento transcorrente (Fig. 7a).

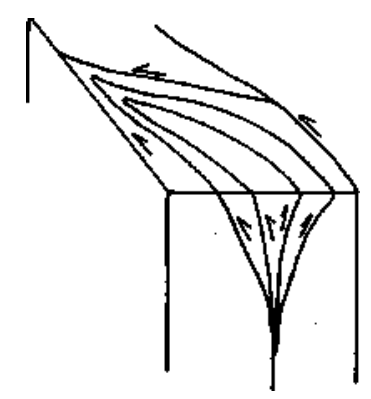

Figura 6 - Bloco diagrama do segmento norte do duplex $\mathrm{Cu}$ ruru. O plano frontal do bloco diagrama corresponde a uma seção NE-SW no duplex, com o observador olhando para noroeste. Sem escala

Figure 6 - Block diagram of the northern segment of the Cururu duplex. The front plan of the block diagram is a NE-SW section in the duplex, with the observer facing northwest. Without scale

História Cinemática As principais estruturas que compõem o Lineamento Cinzento são constituídas por conjuntos de rochas supracrustais atrelados à fase de transtração inicial. A análise de conteúdo litológico mostra que há diferenças expressivas entre as unidades estratigráficas, sugerindo a existência de bacias com características litoestruturais próprias.

Embora seja difícil constatar, no momento, o sincronismo entre as unidades litológicas, em função da ausência de informações geocronológicas de detalhe, sabe-se que as bacias experimentaram inversão durante uma fase de transpressão regional. Nesse período instalaram-se estruturas positivas sob condições da fácies anfibolito em Salobo e xisto verde em Cururu e Serra Pelada. Grande parte das zonas de cisalhamento orientadas nas direções N-S, NNE-SSW, NE-SW e NNW-SSE, com movimentação dextral, deve estar associada a essa fase de movimentação cinemática.

Na progressão da movimentação, a estrutura do Salobo 
$\mathbf{A}$
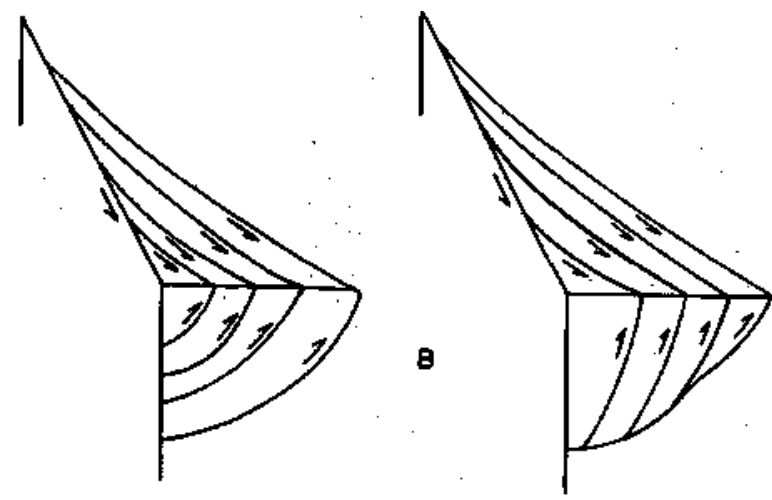

Figura 7 - Blocos diagramas mostrando os modelos geométricos visualizados para o rabo de cavalo Serra Pelada. As zonas de cisalhamento secundárias divergem da superficie de deslocamento transcorrente em A. e do deslocamento com caráter de cavàlgamento em B. Os planos frontais dos blocos diagramas constituem seções NW-SE na parte central do rabo de cavalo, com o observador olhando para sudoeste. Sem escala Figure 7 - Block diagram showing the idealized geometric models of the Serra Pelada horsetail. Minor shear zones diverge from the strikeslip detachment zone (A) and from the thrust zone (B). The frontal planes of the block diagrams are N-S sections in the central part of the horsetail structure, with the observer facing southwest. Without scale

experimentou transtração, em condições térmicas da fácies xisto verde. Dessa época, não há registro de transtração nas demais estruturas, refletindo o caráter heterogêneo da movimentação ao longo do lineamento. A natureza heterogênea dos movimentos no sistema transcorrente é função da interação de faixas de zonas com diferentes intensidades de movimentação e da rotação de blocos irregulares limitados, em parte, pelas zonas de cisalhamento.

A última fase de movimentação cinemática é caracterizada pela instalação de uma zona de cisalhamento $Y$ ou $D$ que secciona e desloca as estruturas do Salobo e Cururu. A essa fase podem estar vinculadas as estruturas em rabo de cavalo observadas nas adjacências da estrutura do Cururu e na extremidade oeste do lineamento.

Portanto, as complexidades geométricas existentes ao longo do Lineamento Cinzento são interpretadas como produtos decorrentes de movimentos cinemáticos distintos. Não se descarta, no entanto, a interpretação que prevê outras fases cinemátícas sem expressão regional.

CONCLUSÕES O Lineamento Cinzento é interpretado como uma faixa transcorrente sinistrai, composta por estruturas com características litoestruturais próprias, formadas a partir da interação de zonas de cisalhamento e de mudanças na orientação das mesmas; ao primeiro caso, associam-se as feições romboédricas e, ao segundo, as estruturas sigmóidais. Sua evolução cinemática envolve várias fases principais de movimentação, daí a complexidade geométrica constatada por ocasião da análise integrada dos diversos elementos estruturais. Algumas estruturas menores, que se superpõem aos arranjos estruturais maiores e promovem complicações adicionais ao quadro geométrico, podem estar ligadas a movimentos cinemáticos de expressão local.

Esse lineamento é também considerado como uma fração particular ligada à evolução do cinturão de cisalhamento Itacaiunas no final do Arqueano. Sabe-se que esse cinturão incorpora movimentação essencialmente oblíqua, desmembrada em componentes de cavàlgamento e direciona!, que resultou na instalação de sistemas imbricados e transcorrentes sinistrais paralelizados.

A expressiva diversidade geométrica ao longo do Lineamento Cinzento e a fácil visualização de suas estruturas nos diferentes produtos de sensores remotos permitem classificá-lo como um exemplo didático, que poderá ajudar na definição de outros sistemas transcorrentes em terrenos pré-cambrianos.

\section{REFERÊNCIAS BIBLIOGRÁFICAS}

ARAÚJO, O.J.B.; MAIA, R.G.N.; JOÃO, X.S.J.; COSTA, J.B.S. 1988. A megaestruturacão arqueana da folha Serra dos Carajás. In CONGR. LATINO AMERICANO DE GEOLOGIA, 7, Belém, 1988. Anais... Belém, SBG. p. 324-338.

BADHAM, J.P.N. 1982 Strike-slip orogens - an explanation for the Hercynides. J. Geol. Soe. London, 139:493-504.

BALANCE, P.P. \& READING, H.G. 1980. Sedimentation in oblique-slip mobile zones. Intern. Assoc. Sediment. 282p. (Sp. Public. 4)

BLICK, N.C. \& BIDDLE, K.T. 1985. Deformation and basin formation along strike-slip faults. In: BLICK, N.C. \& BIDDLE, K.T., eds. Strike-Slip deformation, basin formation and sedimentation. Soc. Econ. Paleont. Mineral. 37:1-34. (Sp. Public.)

CROWELL, J.C. 1974. Origin of Late Cenozoic Basins in Southern California. In: DICKINSON, W.R. eds.. Tectonics and Sedimentation. Soc. Econ. Paleont Mineral. 22:190-204. (Sp. Public.).

DEWEY, J.F. 1982. Plate tectonics and the evolution of the British Isles. /. Geol. Soc. Lond., 139:371-412.

OXBURGH, F.R. 1974. The plain man's guide to plate tectonics. Proc. Geol. Ass., 85:299-357.
SANDERSON, D.J. \& MARCHINI, W.R.D. 1984. Transpression. J. Struct; Geol., 6:449-459.

SOUZA, Z.S.; SANTOS, A.B.; LEDSHAN, EJ ; MARTINS, L.P.B.; HOHN, S.R.B.; COSTA, V.G. 1988. Feições geológicas e estruturais do Greenstone Belt de Identidade, região de Xinguara-Rio Maria, Sul do Pará. In: CONGR. BRÁS. GEOL., 35, Belém, 1988 .Anais... Belém, SBG. v.3, p. 1453-1467.

WILCOX, R.E.; HARDING, T.P.; SEELY, D.R. 1973. Basic wrench tectonics. AAPGButt., 57(1):74-96.

WOODCOK, N.H. \& FISCHER, M. 1986. Strike-slip duplexes. $J$. Struct. Geol. 6(7):72-735.

MANUSCRITO A619 Recebido em 21 de agosto de 1989 Revisão do autor em 17 de abril de 1990 Revisão aceita em 18 de abril de 1990 